\title{
Análise da prevalência de morte súbita e os fatores de riscos associados
}

\author{
Almeida, R.J.O.; Ortiz, E.T.; Weingartner, A.C.; \\ Apresentador: Raquel Joana de Oliveira Almeida
}

\section{Resumo}

Introdução: Morte súbita é definida como morte instantânea ou dentro de 24 horas após início dos sinais e sintomas. Objetivo: Avaliar a prevalência de morte súbita e correlacionar com fatores de riscos associados em necropsias realizadas no Serviço de Verificação de Óbitos (SVO). Material e métodos: Foram analisados os relatórios finais de necropsias do SVO entre 1993 a 2002 e coletados os dados clínicos e a causa do óbito. Resultados: Das 2.056 autópsias do período, 780 (37,93\%) eram de morte súbita. A idade média foi de 61,76 anos, sendo $62,56 \%$ do sexo masculino, com média de idade de 59,45 anos, e mulheres com média de idade de 65,62 anos. A principal causa de morte súbita foi infarto agudo do miocárdio (IAM) (48,71\%). A principal moléstia de base associada foi aterosclerose (80\%). Ao correlacionar a idade com a causa da morte súbita observou-se que a idade média dos pacientes com acidente vascular cerebral (AVC) hemorrágico (52,1 anos) era menor do que de outras causas, como AVC isquêmico (68,3 anos), IAM (62,92 anos) e insuficiência cardíaca crônica (ICC) (62anos) ( $\mathrm{p}<0,001)$. Conclusão: Há mais morte súbita entre homens do que entre mulheres, e essas morrem com idade mais avançada do que os homens. A principal causa relatada como morte súbita foi o IAM. Observou-se, ainda, que os pacientes com AVC hemorrágico morrem mais precocemente do que aqueles que faleceram por outras causas.

\section{Referência:}

Almeida, R.J.O.; Ortiz, E.T.; Weingartner, A.C.;. Análise da prevalência de morte súbita e os fatores de riscos associados. In: II Congresso Brasileiro de Medicina Hospitalar - II CBMH [= Blucher Medical Proceedings, vol.1, num.5] São Paulo: 